\title{
Differences in 25-hydroxy vitamin D and vitamin D-binding protein concentrations according to the severity of endometriosis
}

\author{
Jong Chul Baek', Jae Yoon Jo' ${ }^{2}$, Seon Mi Lee ${ }^{2}$, In Ae Cho ${ }^{2}$, Jeong Kyu Shin ${ }^{2,3,4}$, Soon Ae Lee ${ }^{2,3,4}$, Jong Hak Lee ${ }^{2,3,4}$, Min-Chul Cho ${ }^{4,5}$, \\ Won Jun $\mathrm{Choi}^{2,3,4}$ \\ 'Department of Obstetrics and Gynecology, Gyeongsang National University Changwon Hospital, Changwon; ${ }^{2}$ Department of Obstetrics and \\ Gynecology, Gyeongsang National University Hospital, Jinju; ${ }^{3}$ Department of Obstetrics and Gynecology, Gyeongsang National University College of \\ Medicine, Jinju; ${ }^{4}$ nstitute of Health Sciences, Gyeongsang National University, Jinju; ${ }^{5}$ Department of Laboratory Medicine, Gyeongsang National \\ University Hospital, Gyeongsang National University College of Medicine, Jinju, Korea
}

Objective: To investigate serum 25-hydroxyl vitamin D (25(OH)D) and vitamin D-binding protein (VDBP) concentrations in women with endometriosis according to the severity of disease.

Methods: Women with mild endometriosis $(n=9)$ and advanced endometriosis $(n=7)$, as well as healthy controls $(n=16)$, were enrolled in this observational study. Serum total $25(\mathrm{OH}) \mathrm{D}$ concentrations were analyzed using the Elecsys vitamin D total kit with the Cobas e602 module. Concentrations of bioavailable and free $25(\mathrm{OH}) \mathrm{D}$ were calculated. Concentrations of VDBP were measured using the Human Vitamin D BP Quantikine ELISA kit. Variables were tested for normality and homoscedasticity using the Shapiro-Wilk test and Leven F test, respectively. Correlation analysis was used to identify the variables related to total 25(OH)D and VDBP levels. To assess the effects of total 25(OH)D and VDBP levels in the three groups, multivariate generalized additive modeling (GAM) was performed.

Results: Gravidity and parity were significantly different across the three groups. Erythrocyte sedimentation rate (ESR) and CA-125 levels increased as a function of endometriosis severity, respectively $(p=0.051, p=0.004)$. The correlation analysis showed that total $25(\mathrm{OH}) \mathrm{D}$ levels were positively correlated with gravidity $(r=0.59, p<0.001)$ and parity $(r=0.51, p<0.003)$. Multivariate GAM showed no significant relationship of total 25(OH)D levels with EMT severity after adjusting for gravidity and ESR. However, the coefficient of total 25(OH)D levels with gravidity was significant $(1.87 ; 95 \%$ confidence interval, $0.12-3.63 ; p=0.040)$.

Conclusion: These results indicate that vitamin D and VDBP levels were not associated with the severity of endometriosis.

Keywords: Endometriosis; Vitamin D; Vitamin D-binding protein

\section{Introduction}

Several theories have been presented regarding the pathogenesis

Received: Oct 21, 2018 · Revised: May 6, 2019 · Accepted: May 10, 2019 Corresponding author: Won Jun Choi

Department of Obstetrics and Gynecology, Gyeongsang National University Hospital, Gyeongsang National University College of Medicine, 79 Gangnam-ro, Jinju 52727, Korea

Tel: +82-55-750-8147 Fax:+82-55-759-1118 E-mail:choiwj@gnu.ac.kr

This is an Open Access article distributed under the terms of the Creative Commons Attribution Non-Commercial License (http://creativecommons.org/licenses/by-nc/4.0/) which permits unrestricted non-commercial use, distribution, and reproduction in any medium, provided the original work is properly cited. of endometriosis; however, to date, the etiology and factors potentially involved in its development and progression remain poorly understood. Some authors have theorized that endometriosis should be considered a multifactorial disease involving genetic, hormonal, immunological, and environmental factors [1]. In particular, inflammatory processes are thought to play an important role in the development and progression of endometriosis, as abnormal levels of proinflammatory cytokines have been observed in the peritoneal fluid and serum of women with endometriosis [2].

Vitamin D is considered to be an important modulator of the immune system, and the potential role of vitamin $D$ in endometriosis is 
of significant interest [3]. In particular, in vitro studies have demonstrated the anti-inflammatory, anti-proliferative, and anti-invasive effects of vitamin $D$ in several immune-related pathologies, including rheumatoid arthritis [4], psoriasis [5], and cancer [6]. Evidence for a role of vitamin $D$ in the pathogenesis of endometriosis is also beginning to emerge.

In the circulation, the majority of 25-hydroxyl vitamin D (25(OH)D) is bound to vitamin D-binding protein (VDBP). VDBP plays a major role in maintaining total and free $25(\mathrm{OH}) \mathrm{D}$ concentrations. Albumin is also a low-affinity carrier of $25(\mathrm{OH}) \mathrm{D}$, which increases tissue availability. According to the free hormone hypothesis, only unbound vitamin $\mathrm{D}$ is physiologically active [7]; however, plasma total $25(\mathrm{OH}) \mathrm{D}$ concentrations, not free $25(\mathrm{OH}) \mathrm{D}$ levels, are used to determine vitamin $D$ status, as free or unbound $25(\mathrm{OH}) \mathrm{D}$ comprises less than $0.1 \%$ of total $25(\mathrm{OH}) \mathrm{D}$. Bioavailable $25(\mathrm{OH}) \mathrm{D}$ is defined as the combination of free and albumin-bound $25(\mathrm{OH}) \mathrm{D}$ or $25(\mathrm{OH}) \mathrm{D}$ that is not bound to VDBP, and represents up to $10 \%$ of total $25(\mathrm{OH}) \mathrm{D}$ [7]. In addition to transporting $25(\mathrm{OH}) \mathrm{D}$, VDBP is also crucial for the conversion of $25(\mathrm{OH}) \mathrm{D}$ to its active form, 1,25(OH)D, and for the reabsorption of 25(OH)D metabolites in the kidneys. VDBP also has been shown to act as an actin scavenger, macrophage activator, and inflammatory marker [8], and VDBP levels have been found to vary by the degree of insulin resistance, liver and kidney disease, pregnancy, and inflammation [9].

Because $25(\mathrm{OH}) \mathrm{D}$ has been suggested to play an important pathological role in endometriosis, we hypothesized that serum concentrations of 25(OH)D and VDBP would vary with the severity of the disease. The aim of this study was to investigate $25(\mathrm{OH}) \mathrm{D}$ status via total $25(\mathrm{OH}) \mathrm{D}$, bioavailable and free $25(\mathrm{OH}) \mathrm{D}$, and serum VDBP levels in women with mild and advanced endometriosis.

\section{Methods}

\section{Study participants}

All participants in this study were patients admitted to Gyeongsang National University Hospital, Jinju, Korea from February 2017 to May 2018. All patients underwent laparoscopy to stage the severity of their endometriosis according to the Revised Classification of the American Society for Reproductive Medicine (ASRM) [10]. All patients were differentially diagnosed and staged based on a pathological review of the surgical specimens.

Patients in this study were divided into three groups: mild endometriosis (ASRM stage I/II), advanced endometriosis (ASRM stage III/IV), and healthy controls. Healthy controls were recruited from women who were at the facility for a health screening and did not report any symptoms of endometriosis. Whole-blood and serum samples were collected from nine patients with mild endometriosis, seven patients with advanced endometriosis, and 16 healthy controls.

This study was approved by the Institutional Review Board of the Gyeongsang National University Hospital (IRB No. GNUH 2017-01004).

\section{Serum total 25(OH)D and VDBP concentrations}

Each serum sample was aliquoted into two tubes and stored at $-80^{\circ} \mathrm{C}$ until it could be analyzed for total 25(OH)D and VDBP levels. Serum total $25(\mathrm{OH}) \mathrm{D}$ concentrations were analyzed using the Elecsys vitamin D total kit with the Cobas e602 module (Roche Diagnostics, Mannheim, Germany), which is an electrochemiluminescent assay with ruthenium-labeled VDBP, biotin-labeled vitamin $\mathrm{D}$, and streptavidin-coated microparticles. VDBP concentrations were measured using the Human Vitamin D BP Quantikine ELISA kit (R\&D Systems, Minneapolis, MN, USA) according to the manufacturer's protocol.

\section{Calculation of bioavailable and free 25(OH)D}

Concentrations of bioavailable and free 25(OH)D were calculated from the total $25(\mathrm{OH}) \mathrm{D}, \mathrm{VDBP}$, and serum albumin concentrations using the following equations $[11,12]$.

Bioavailable 25(OH)D = albumin-bound 25(OH)D+free 25(OH)D

$$
=\left(\text { albumin } \times K_{\text {albumin }}+1\right) \times \text { free } 25(\mathrm{OH}) \mathrm{D}
$$

Free $25(\mathrm{OH}) \mathrm{D}=\frac{-b+\sqrt{\mathrm{b}^{2}-4 a c}}{2 \mathrm{a}}$

$\mathrm{a}=K_{V D B P} \times K_{\text {albumin }} \times$ albumin $+K_{V D B P}$

$\mathrm{b}=K_{V D B P} \times \mathrm{VDBP}-K_{V D B P} \times$ total $25(\mathrm{OH}) \mathrm{D}+K_{\text {allumin }} \times$ albumin +1

$\mathrm{c}=-$ total $25(\mathrm{OH}) \mathrm{D}$

$K_{V D B P}[$ for genotype-constant bioavailable $25(\mathrm{OH}) \mathrm{D}]=0.7 \times 10^{9} \mathrm{M}^{-1}$

To calculate genotype-specific bioavailable $25(\mathrm{OH}) \mathrm{D}$ levels, the GC genotype-specific VDBP binding affinity $\left(K_{V D B P I f}, 1.12 \times 10^{9} \mathrm{M}^{-1} ; K_{V D B P} I_{\text {s, }}\right.$ $\left.0.6 \times 10^{9} \mathrm{M}^{-1} ; K_{V D B P 2}, 0.36 \times 10^{9} \mathrm{M}^{-1}\right)$ was used instead of the generic $K_{V D B P}[13]$. For heterozygous genotypes, the mean affinity for the two homozygotes was used (KVDPPI//s, $0.86 \times 10^{9} \mathrm{M}^{-1} ; K_{\text {VDBP } 1 / 2,2}, 0.74 \times 10^{9}$ $\left.\mathrm{M}^{-1} ; K_{V D B P 1 / 2 / 2}, 0.48 \times 10^{9} \mathrm{M}^{-1}\right)$ [14]. In this study, bioavailable $25(\mathrm{OH}) \mathrm{D}$ referred to both genotype-constant and genotype-specific bioavailable 25(OH)D.

\section{Statistical analysis}

Continuous variables were presented as mean with standard deviation and median with interquartile range. The variables were tested for normality and homoscedasticity using the Shapiro-Wilk test and Leven $\mathrm{F}$ test, respectively. If variables satisfied normality and homoscedasticity, analysis of variance was used to compare the values among the three groups. Otherwise, the Kruskal-Wallis test was used. The Fisher exact test was used to compare the proportions of categorical variables by groups.

Correlation analysis was used to identify variables related to total 
25(OH)D and VDBP levels. If the normality assumption was satisfied, Pearson correlation analysis was performed, and if not, Spearman correlation analysis was used.

Multivariate generalized additive modeling (GAM) was performed to identify the effects of total $25(\mathrm{OH}) \mathrm{D}$ and VDBP in the three groups. The variables included in the multivariate analysis were those that showed an association with total 25(OH)D or VDBP levels in the correlation analysis, with a $p$-value of 0.20 or less. Among the variables associated with total 25(OH)D and VDBP, if the correlation coefficient between the variables was more than 0.7 , only one variable was included in the multivariate analysis to avoid the problem of multicollinearity. All analyses were conducted using R ver. 3.52 (R Foundation, Vienna, Austria). A two-sided test $p$-value $<0.05$ was considered to indicate statistical significance.

Table 1. Demographic and clinical factors of participants by group

\begin{tabular}{|c|c|c|c|c|}
\hline Variable & Healthy control $(n=16)$ & Mild EMT $(n=9)$ & Advanced EMT $(n=7)$ & $p$-value ${ }^{a)}$ \\
\hline Age (yr) & & & & 0.304 \\
\hline Mean \pm SD & $37.31 \pm 6.35$ & $35.44 \pm 7.20$ & $32.57 \pm 6.95$ & \\
\hline Median (IQR) & $38.50(32.00-42.25)$ & $36.00(32.00-42.00)$ & $32.00(29.00-36.50)$ & \\
\hline BMI $\left(\mathrm{kg} / \mathrm{m}^{2}\right)$ & & & & 0.236 \\
\hline Mean $\pm S D$ & $21.69 \pm 2.29$ & $21.28 \pm 2.09$ & $23.77 \pm 2.92$ & \\
\hline Median (IQR) & 21.50 (19.93-23.75) & $21.80(20.00-22.90)$ & $23.70(22.75-24.35)$ & \\
\hline Gravidity & & & & 0.001 \\
\hline Mean \pm SD & $2.62 \pm 1.09$ & $1.22 \pm 1.09$ & $0.57 \pm 0.79$ & \\
\hline Median (IQR) & $2.50(2.00-3.00)$ & $1.00(0.00-2.00)$ & $0.00(0.00-1.00)$ & \\
\hline Parity & & & & 0.002 \\
\hline Mean \pm SD & $1.88 \pm 0.72$ & $1.11 \pm 0.93$ & $0.43 \pm 0.53$ & \\
\hline Median (IQR) & $2.00(1.00-2.00)$ & $1.00(0.00-2.00)$ & $0.00(0.00-1.00)$ & \\
\hline Place of residence, $n(\%)$ & & & & 0.882 \\
\hline Urban & $12(52.2)$ & $6(26.1)$ & $5(21.7)$ & \\
\hline Rural & $4(44.4)$ & $3(33.3)$ & $2(22.2)$ & \\
\hline Albumin (mg/dL) & & & & 0.935 \\
\hline Mean \pm SD & $4.66 \pm 0.29$ & $4.63 \pm 0.26$ & $4.60 \pm 0.29$ & \\
\hline Median (IQR) & $4.70(4.40-4.90)$ & $4.60(4.40-4.80)$ & $4.60(4.45-4.85)$ & \\
\hline $\mathrm{ESR}(\mathrm{mm} / \mathrm{hr})$ & & & & 0.051 \\
\hline Mean \pm SD & $10.70 \pm 8.26$ & $15.76 \pm 11.97$ & $24.70 \pm 12.02$ & \\
\hline Median (IQR) & $8.75(4.17-14.43)$ & $14.00(7.00-27.00)$ & $29.00(20.95-31.00)$ & \\
\hline $\mathrm{CRP}(\mathrm{mg} / \mathrm{L})$ & & & & 0.841 \\
\hline Mean \pm SD & $0.78 \pm 1.31$ & $3.52 \pm 7.62$ & $2.30 \pm 4.46$ & \\
\hline Median (IQR) & $0.20(0.10-0.72)$ & $0.20(0.10-0.50)$ & $0.40(1.10-1.55)$ & \\
\hline CA-125 (IU/mL) & & & & 0.004 \\
\hline Mean $\pm S D$ & $23.87 \pm 16.21$ & $192.22 \pm 335.01$ & $140.97 \pm 137.62$ & \\
\hline Median (IQR) & $20.30(13.97-27.10)$ & $39.40(23.50-161.00)$ & $102.00(41.90-182.45)$ & \\
\hline Total 25(OH)D (ng/mL) & & & & 0.006 \\
\hline Mean \pm SD & $16.96 \pm 4.71$ & $14.17 \pm 7.62$ & $8.91 \pm 1.67$ & \\
\hline Median (IQR) & $18.09(13.60-18.64)$ & $12.50(9.70-21.30)$ & $9.30(8.45-9.40)$ & \\
\hline Bioavailable $25(\mathrm{OH}) \mathrm{D}(\mathrm{ng} / \mathrm{mL})$ & & & & 0.062 \\
\hline Mean \pm SD & $1.95 \pm 0.79$ & $2.45 \pm 1.36$ & $1.30 \pm 0.35$ & \\
\hline Median (IQR) & $1.75(1.53-2.00)$ & $2.67(1.36-3.08)$ & $1.22(1.07-1.53)$ & \\
\hline Free $25(\mathrm{OH}) \mathrm{D}(\mathrm{ng} / \mathrm{mL})$ & & & & 0.085 \\
\hline Mean \pm SD & $4.81 \pm 1.74$ & $6.30 \pm 3.28$ & $3.36 \pm 1.00$ & \\
\hline Median (IQR) & $4.32(3.94-5.03)$ & $7.15(3.40-8.51)$ & $3.29(2.51-4.27)$ & \\
\hline $\operatorname{VDBP}(\mathrm{ng} / \mathrm{mL})$ & & & & 0.241 \\
\hline Mean $\pm S D$ & $169.20 \pm 36.31$ & $161.25 \pm 53.09$ & $198.34 \pm 42.54$ & \\
\hline Median (IQR) & 169.77 (142.02-196.08) & $156.05(124.05-179.57)$ & 205.47 (173.09-218.44) & \\
\hline
\end{tabular}

EMT, endometriosis (mild, American Society for Reproductive Medicine [ASRM] stage I and II; advanced, ASRM stage III and IV); SD, standard deviation; IQR, interquartile range; $\mathrm{BMI}$, body mass index; $\mathrm{ESR}$, erythrocyte sedimentation rate; $C R P$, C-reactive protein; $25(\mathrm{OH}) \mathrm{D}, 25$-hydroxyl vitamin D; VDBP, vitamin D-binding protein.

${ }^{\text {a) }} p$-value $<0.05$ is considered to indicate statistical significance. 
Table 2. Correlations between demographic and clinical factors and total 25(OH)D and VDBP levels

\begin{tabular}{lcccc}
\hline \multirow{2}{*}{ Variable } & \multicolumn{2}{c}{ Total 25(OH)D } & & VDBP \\
\cline { 2 - 3 } \cline { 5 - 5 } & Correlation coefficient & $p$-value & Correlation coefficient & $p$-value \\
\hline Age & 0.21 & 0.24 & -0.34 & 0.056 \\
BMI & -0.13 & 0.467 & 0.12 & 0.531 \\
Gravity & 0.59 & $<0.001$ & -0.13 & 0.481 \\
Parity & 0.51 & 0.003 & -0.23 & 0.209 \\
Place & & 0.63 & & 0.869 \\
Urban & $13.27(9.35-18.41)$ & & $162.81(146.16-210.54)$ & $176.80(134.80-186.80)$ \\
Rural & $13.71(9.70-18.50)$ & & -0.14 & 0.439 \\
Albumin & -0.07 & 0.7 & 0.34 & 0.055 \\
ESR & -0.32 & 0.071 & 0.34 & 0.055 \\
CRP & -0.32 & 0.071 & 0.14 & 0.448 \\
CA-125 & -0.06 & 0.724 & & \\
VDBP & 0.03 & 0.077 & & \\
\hline
\end{tabular}

25(OH)D, 25-hydroxyl vitamin D; VDBP, vitamin D-binding protein; BMI, body mass index; ESR, erythrocyte sedimentation rate; CRP, C-reactive protein.

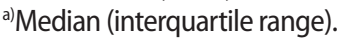

Table 3. Multivariate generalized additive modeling for total 25(OH)D and VDBP levels

\begin{tabular}{|c|c|c|c|c|}
\hline \multirow{2}{*}{ Variable } & \multicolumn{2}{|c|}{ Total 25(OH)D } & \multicolumn{2}{|c|}{ VDBP } \\
\hline & Regression coefficient $(95 \% \mathrm{Cl})$ & $p$-value & Regression coefficient $(95 \% \mathrm{Cl})$ & $p$-value \\
\hline \multicolumn{5}{|l|}{ Group } \\
\hline Healthy control & 0.00 & - & 0.00 & - \\
\hline Mild EMT & 0.20 (-4.68 to 5.07$)$ & 0.935 & $-9.17(-52.82$ to 34.49$)$ & 0.672 \\
\hline Advanced EMT & $-2.46(-8.82$ to 3.90$)$ & 0.437 & 23.8 (-34.64 to 82.25$)$ & 0.414 \\
\hline Age & & & $-2.45(-5.79$ to -0.9$)$ & 0.151 \\
\hline Gravidity & $1.87(0.12$ to 3.63$)$ & 0.040 & & \\
\hline Parity & & & $10.69(-19.3$ to 40.68$)$ & 0.474 \\
\hline ESR & $-0.12(-0.30$ to 0.06$)$ & 0.177 & $0.81(-0.87$ to 2.49$)$ & 0.337 \\
\hline
\end{tabular}

25(OH)D, 25-hydroxyl vitamin D; VDBP, vitamin D-binding protein; Cl, confidence interval; EMT, endometriosis (mild, American Society for Reproductive Medicine [ASRM] stage I and II; advanced, ASRM stage III and IV); ESR, erythrocyte sedimentation rate.

\section{Results}

Table 1 shows the clinical characteristics of the study participants according to the severity of endometriosis. Gravidity $(p=0.001)$ and parity $(p=0.002)$ were significantly different across the three groups. There was no significant difference in age or place of residence (as a proxy for sunlight exposure) across the three groups.

The laboratory findings of the study participants are reported in Table 1. There were no significant differences in body mass index or serum albumin levels. The erythrocyte sedimentation rate (ESR), as an index of inflammation, increased as a function of endometriosis severity $(p=0.051)$. C-reactive protein, another inflammatory marker, was not significantly different across the three groups. In addition, serum CA-125 levels were significantly different across the three groups ( $p=0.004$ ) with higher levels observed in both groups with endometriosis than in healthy controls.

To confirm the relationships of total 25(OH)D and VDBP with other variables, correlation analysis was performed. The results showed that total $25(\mathrm{OH}) \mathrm{D}$ levels were positively correlated with gravidity $(r=0.59, p<0.001)$ and parity $(r=0.51, p=0.003)$, but not with ESR $(r=-0.32, p=0.071)$ and CA-125 ( $r=-0.06, p=0.724)$ (Table 2).

Multivariate GAM showed no significant relationship of vitamin D levels with endometriosis severity after adjusting for gravidity and ESR. However, total 25(OH)D levels were significantly correlated with gravidity $(1.87 ; 95 \%$ confidence interval $[\mathrm{Cl}], 0.12-3.63 ; p=0.040)$. VDBP showed no significant correlations with other variables (Table 3 ). In pairwise comparisons, serum levels of total $25(\mathrm{OH}) \mathrm{D}$ tended to show a negative relationship with the severity of endometriosis, but this relationship was not statistically significant across the three groups. Serum levels of bioavailable and free 25(OH)D did not differ according to the severity of endometriosis. However, the serum levels of total, bioavailable, and free $25(\mathrm{OH}) \mathrm{D}$ in the advanced endometriosis group were significantly lower than in the healthy control group ( $p=0.001, p=0.018$, and $p=0.049$, respectively). For serum 

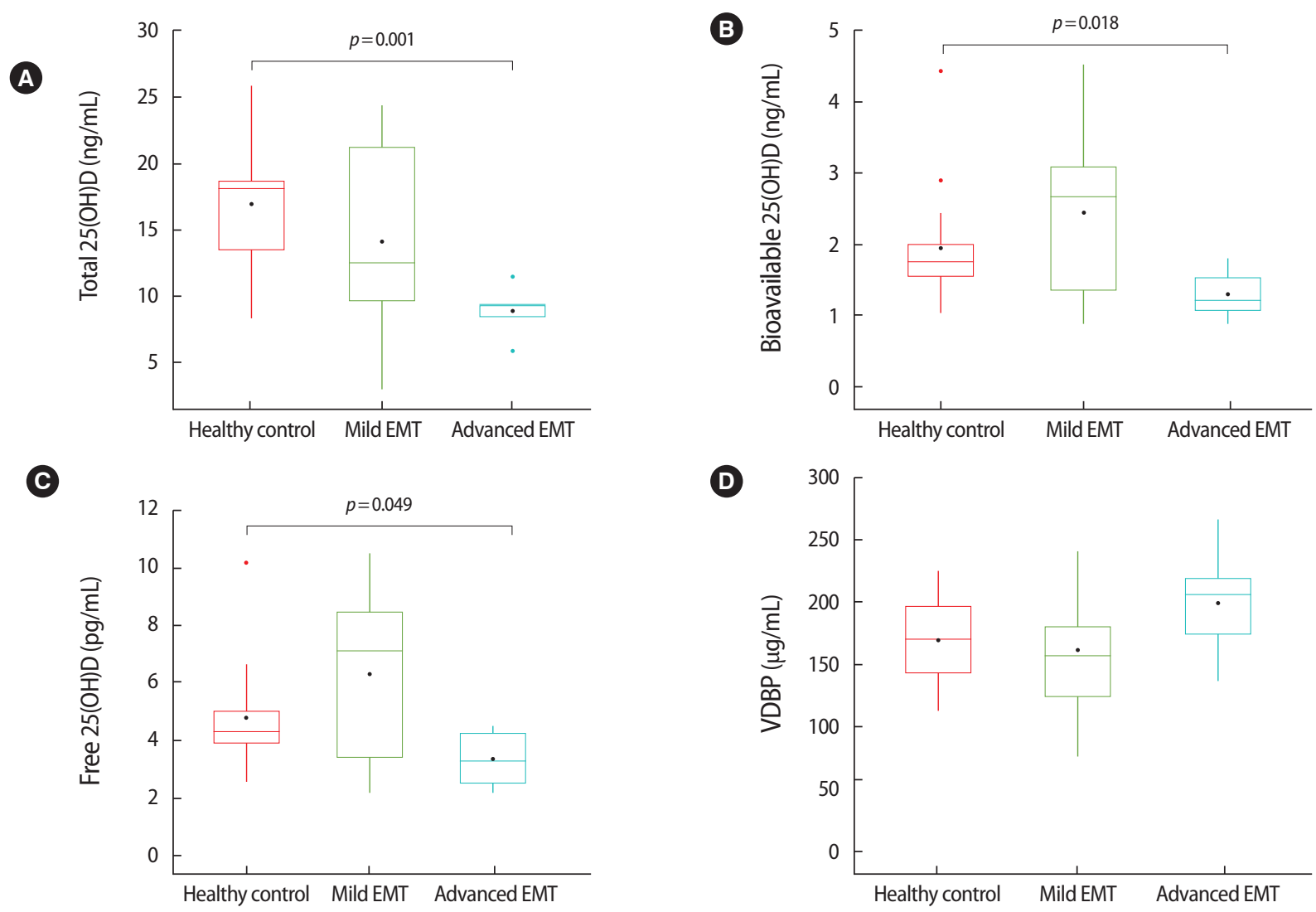

Figure 1. Pairwise comparison of serum $(A)$ total, $(B)$ bioavailable, $(C)$ free 25 -hydroxyl vitamin $D(25(O H) D)$, and (D) vitamin $D$-binding protein (VDBP) according to disease severity. EMT, endometriosis (mild, American Society for Reproductive Medicine [ASRM] stage I and II; advanced, ASRM stage III and IV).

concentrations of VDBP, we found no significant differences between the healthy control group and either group defined in terms of endometriosis severity (Figure 1).

\section{Discussion}

As in many chronic diseases, vitamin D has long been proposed as an important modulator in the pathogenesis of endometriosis, but this possibility remains controversial and is under investigation [15]. Vitamin D also plays a role in the regulation of sex hormone steroidogenesis. In addition, increasing evidence suggests that $25(\mathrm{OH}) \mathrm{D}$ may play a regulatory role in symptoms associated with polycystic ovary syndrome. Vitamin D deficiency has been reported to contribute to the pathogenesis of endometriosis via its immunomodulatory and anti-inflammatory properties [16].

Previous studies that have investigated serum 25(OH)D levels according to the presence or absence of endometriosis have reported inconsistent findings. One study showed lower levels of 25(OH)D in patients with endometriosis [17]. A study by Harris et al. of women enrolled in the Nurses' Health Study II reported that predicted plasma
25(OH)D levels were inversely associated with endometriosis risk; the authors of that study recommended consumption of foods high in 25(OH)D to prevent endometriosis [18]. In contrast, other studies have reported nonsignificant elevations in $25(\mathrm{OH}) \mathrm{D}$ among women with endometriosis $[19,20]$. Prior studies measured total 25(OH)D concentrations, but did not consider bioavailable or free $25(\mathrm{OH}) \mathrm{D}$. Vitamin D also is known to show varying activity depending on the degree of binding with VDBP. To the best of our knowledge, few studies have investigated bioavailable and free $25(\mathrm{OH}) \mathrm{D}$ and VDBP according to the severity of endometriosis. Our study compared total, bioavailable, and free $25(\mathrm{OH}) \mathrm{D}$ concentrations, as well as VDBP concentrations, between women with mild and advanced endometriosis and healthy controls. In our study, total 25(OH)D tended to show an inverse relationship with the severity of the disease. This finding is consistent with a previous study that reported significantly lower total $25(\mathrm{OH}) \mathrm{D}$ concentrations in women with severe endometriosis than in both the healthy controls and in women with mild endometriosis [21].

An analysis of the clinical characteristics of patients enrolled in this study indicated that age and BMI did not differ across the three groups. We found that the number of pregnancies and the number 
of births were significantly lower in patients with more severe disease. This finding suggests that infertility is more common in patients with endometriosis, particularly in those with severe endometriosis. However, the exact relationship between endometriosis and infertility remains unclear [22].

In our study, ESR and CA-125 concentrations were higher in the endometriosis groups. ESR was different across the three groups in our study, and showed a significant tendency to increase with greater disease severity. This finding may be related to the varying extent of tissue destruction that occurs as endometriosis progresses. Our finding is consistent with a meta-analysis of CA-125 levels in the diagnosis of endometriosis, which suggested that serum CA-125 may be a useful biomarker for the noninvasive diagnosis of endometriosis [23]. However, meaningful correlations of total $25(\mathrm{OH}) \mathrm{D}$ levels with the ESR and CA-125 were not found. The correlation analysis showed that total $25(\mathrm{OH}) \mathrm{D}$ levels were positively correlated with gravidity and parity, but not with ESR and CA-125 (Table 2).

To investigate the effects of serum levels of total 25(OH)D and VDBP on the severity of endometriosis, we performed multivariate GAM. After adjusting for gravidity and ESR, total 25(OH)D and VDBP levels were not significantly related with endometriosis severity. However, total $25(\mathrm{OH}) \mathrm{D}$ levels were significantly correlated with gravidity. Our results suggest that serum concentration of total $25(\mathrm{OH}) \mathrm{D}$ was related to the number of births regardless of the concentration of VDBP. A number of articles have shown a relationship between childbirth and bone mineral density (BMD), although another report suggested that there is no association between the number of children and BMD. Another study suggested that BMD may be reduced by having a large number of children and a long breastfeeding period [24]. In the present study, higher serum total 25(OH)D levels were associated with higher gravidity. For this reason, the authors suggest that vitamin D may have increased to compensate for lower BMD. The reason for this should be studied in the future research.

A limitation of our study was the small sample of enrolled patients in each group. Our study also did not account for sunlight exposure. We also did not follow up with patients after treatment, which would have helped to clarify etiologic factors in their disease. In conclusion, these results indicate that vitamin D and VDBP levels were not associated with the severity of endometriosis.

\section{Conflict of interest}

No potential conflict of interest relevant to this article was reported.

\section{Acknowledgments}

The authors gratefully acknowledge the enrolled women, the at- tending operative room staff, Eun Seon Sim (RN, a dedicated research nurse), and Dr. Jae Ik Lee (a research assistant).

\section{ORCID}

$\begin{array}{ll}\text { Jong Chul Baek } & \text { https://orcid.org/0000-0003-0919-0800 } \\ \text { Jae Yoon Jo } & \text { https://orcid.org/0000-0001-6499-9085 } \\ \text { Seon Mi Lee } & \text { https://orcid.org/0000-0003-2388-0111 } \\ \text { In Ae Cho } & \text { https://orcid.org/0000-0002-6295-4059 } \\ \text { Jeong Kyu Shin } & \text { https://orcid.org/0000-0001-9050-0874 } \\ \text { Soon Ae Lee } & \text { https://orcid.org/0000-0002-8141-5595 } \\ \text { Jong Hak Lee } & \text { https://orcid.org/0000-0003-3853-0560 } \\ \text { Min-Chul Cho } & \text { https://orcid.org/0000-0002-0609-7734 } \\ \text { Won Jun Choi } & \text { https://orcid.org/0000-0002-4887-3201 }\end{array}$

\section{Author contributions}

Conceptualization: JCB, WJC. Data curation: JYJ, SML, IAC, JKS. Formal analysis: MCC, WJC. Methodology: MCC, WJC. Project administration: WJC. Visualization: SAL, JHL. Writing - original draft: JCB. Writing - review \& editing:WJC.

\section{References}

1. Christodoulakos G, Augoulea A, Lambrinoudaki I, Sioulas V, Creatsas $G$. Pathogenesis of endometriosis: the role of defective' immunosurveillance.' Eur J Contracept Reprod Health Care 2007;12: 194-202.

2. Akoum A, Kong J, Metz C, Beaumont MC. Spontaneous and stimulated secretion of monocyte chemotactic protein-1 and macrophage migration inhibitory factor by peritoneal macrophages in women with and without endometriosis. Fertil Steril 2002;77:989-94.

3. Holick MF. Sunlight and vitamin D for bone health and prevention of autoimmune diseases, cancers, and cardiovascular disease. Am J Clin Nutr 2004;80(6 Suppl):1678S-1688S.

4. van Hamburg JP, Asmawidjaja PS, Davelaar N, Mus AM, Cornelissen $F$, van Leeuwen JP, et al. TNF blockade requires 1,25(OH)2D3 to control human Th17-mediated synovial inflammation. Ann Rheum Dis 2012;71:606-12.

5. Karthaus N, van Spriel AB, Looman MW, Chen S, Spilgies LM, Lieben $\mathrm{L}$, et al. Vitamin $\mathrm{D}$ controls murine and human plasmacytoid dendritic cell function. J Invest Dermatol 2014;134:1255-64.

6. Krishnan AV, Swami S, Peng L, Wang J, Moreno J, Feldman D. Tissue-selective regulation of aromatase expression by calcitriol: implications for breast cancer therapy. Endocrinology 2010;151: 32-42. 
7. Chun RF, Peercy BE, Orwoll ES, Nielson CM, Adams JS, Hewison M. Vitamin D and DBP: the free hormone hypothesis revisited. J Steroid Biochem Mol Biol 2014;144 Pt A:132-7.

8. Chun RF. New perspectives on the vitamin D binding protein. Cell Biochem Funct 2012;30:445-56.

9. Yousefzadeh P, Shapses SA, Wang X. Vitamin D binding protein impact on 25-hydroxyvitamin D levels under different physiologic and pathologic conditions. Int J Endocrinol 2014;2014: 981581.

10. Revised American Society for Reproductive Medicine classification of endometriosis: 1996. Fertil Steril 1997;67:817-21.

11. Bikle DD, Gee E, Halloran B, Kowalski MA, Ryzen E, Haddad JG. Assessment of the free fraction of 25 -hydroxyvitamin $D$ in serum and its regulation by albumin and the vitamin D-binding protein. J Clin Endocrinol Metab 1986;63:954-9.

12. Heijboer AC, Blankenstein MA, Kema IP, Buijs MM. Accuracy of 6 routine 25 -hydroxyvitamin $D$ assays: influence of vitamin $D$ binding protein concentration. Clin Chem 2012;58:543-8.

13. Arnaud J, Constans J. Affinity differences for vitamin D metabolites associated with the genetic isoforms of the human serum carrier protein (DBP). Hum Genet 1993;92:183-8.

14. Song M, Konijeti GG, Yuan C, Ananthakrishnan AN, Ogino S, Fuchs CS, et al. Plasma 25-hydroxyvitamin D, vitamin D binding protein, and risk of colorectal cancer in the nurses' health study. Cancer Prev Res (Phila) 2016;9:664-72.

15. Muscogiuri G, Altieri B, Annweiler C, Balercia G, Pal HB, Boucher $B J$, et al. Vitamin D and chronic diseases: the current state of the art. Arch Toxicol 2017;91:97-107.
16. Muscogiuri G, Altieri B, de Angelis C, Palomba S, Pivonello R, Colao $A$, et al. Shedding new light on female fertility: the role of vitamin D. Rev Endocr Metab Disord 2017; 18:273-83.

17. Anastasi E, Fuggetta E, De Vito C, Migliara G, Viggiani V, Manganaro $\mathrm{L}$, et al. Low levels of $25-\mathrm{OH}$ vitamin $\mathrm{D}$ in women with endometriosis and associated pelvic pain. Clin Chem Lab Med 2017;55:e282-4.

18. Harris HR, Chavarro JE, Malspeis S, Willett WC, Missmer SA. Dairyfood, calcium, magnesium, and vitamin D intake and endometriosis: a prospective cohort study. Am J Epidemiol 2013;177: 420-30.

19. Hartwell D, Rodbro P, Jensen SB, Thomsen K, Christiansen C. Vitamin D metabolites--relation to age, menopause and endometriosis. Scand J Clin Lab Invest 1990;50:115-21.

20. Somigliana E, Panina-Bordignon P, Murone S, Di Lucia P, Vercellini $P$, Vigano $P$. Vitamin D reserve is higher in women with endometriosis. Hum Reprod 2007;22:2273-8.

21. Miyashita M, Koga K, Izumi G, Sue F, Makabe T, Taguchi A, et al. Effects of 1,25-dihydroxy vitamin D3 on endometriosis. J Clin Endocrinol Metab 2016;101:2371-9.

22. de Ziegler D, Borghese B, Chapron C. Endometriosis and infertility: pathophysiology and management. Lancet 2010;376:730-8.

23. Shen A, Xu S, Ma Y, Guo H, Li C, Yang C, et al. Diagnostic value of serum CA125, CA19-9 and CA15-3 in endometriosis: a metaanalysis. J Int Med Res 2015;43:599-609.

24. Mgodi NM, Kelly C, Gati B, Greenspan S, Dai JY, Bragg V, et al. Factors associated with bone mineral density in healthy African women. Arch Osteoporos 2015;10:206. 